\title{
Complementary and alternative medicine (CAM) in acne vulgaris.
}

\author{
Ishmeet Kaur* \\ Department of Dermatology \& STD, University College of Medical Sciences \& GTBH, New Delhi, India
}

Accepted March 20, 2018

\section{Editorial}

Acne vulgaris is a chronic inflammatory disease of pilosabaceous unit which presents with polymorphic lesions like comedones, papules, pustules and nodulocystic lesions. It is prevalent in $80 \%$ of adolescents and $2 / 3^{\text {rd }}$ of adults. Its burden is compounded by its psychological outcomes on the patients with stress, anxiety and depression. Western medicine has provided with a number of effective drugs for the same including benzoyl peroxide, topical and systemic retinoids and antibiotics, dapsone, physical modalities like laser, light therapy and chemical peels. However, over the recent years, despite the success of these standard modalities, there is an increasing proportion of the population trying to explore complementary and alternative medicine (CAM) due to their concerns with cost and adverse effects of the conventional ones such as teratogenicity, photosensitivity, dryness, irritation, acne flare, antibiotic resistance and lack of response and relapse after withdrawal. There are a number of unconventional modalities that have been developed in different aspects of medicine [1-3].

Traditional Chinese Medicine (TCM) including herbal medicine, facial mask, diet modification, acupuncture and face cupping is in a growing demand among many patients of acne. TCM mainly relies on its principle that acne is caused due to increased heat and dampness in the body. The selection of the components of the medicine depends on the basic cause of acne i.e. syndrome of blood heat due to lung heat, syndrome of dampness-heat of spleen and stomach, syndrome of dampnessheat of liver and gallbladder, syndrome of heat-toxin stagnated, syndrome of blood stasis and coagulated phlegm.

Herbal medicine and facial masks are quite popular, mostly due to less cost and a common belief that medicines made out of natural herbs have lesser risk of side effects. These herbs include Phellodendron amurense, gingseng, astragulus, burdock root, scuttellaria (huang qin), licorice, calendula, etc mostly due to their antioxidant, heat clearing and blood purifying action. If lesions are associated with pus, chuan shan jia (Squama manis) or zao jiao ci (Spina gleditsiae) can be used. If associated with resistant cystic lesions herbs like san leng (Rhizoma sparganii) or xia ku cao (Spica prunellae vulgaris) may be added in the face mask. Dietary modification in TCM is based on the principle that certain food products have higher heat component and are proinflammatory such as ginger root, black beans, cinnamon bark, walnut, butter, chicken, lamb, etc. While food items like apple, banana, citrus fruits, broccoli, cauliflower should be encouraged due to their heat clearing and anti- inflammatory property [4-6].

Acupuncture is another popular modality in TCM commonly used for pain management, which is now being successfully applied for treatment of acne vulgaris. In this procedure, needles are inserted into the classic acupoints and properly twisted, which acts on the channels of Qi energy. This Qi energy is believed to be an energy that flows through the body, to every organ and cell, and is critical for health. The human body is connected through channels of Qi energy called meridians, and when these channels are inhibited, it reflects on the skin with acne. Acupuncture helps unblock these channels and is also found to increase the release of nitric oxide in the blood, relieve heat toxicity and improve immunological function. It is also known to balance the androgen levels and decrease the sebum production. Various studies have tried acupoint stimulation technique-such as acupuncture, moxibustion, cupping, acupoint injection, and acupoint catgut embedding for treatment of moderate acne vulgaris and has shown to be effective with reduction of inflammatory lesions. However, still large scale studies are required for proper and reliable evidence $[7,8]$.

Ayurveda is the ancient science of life, vastly followed in India. Mukshadushika is the term given to acne in Sanskrit. Indian systems of medicine like Unani and Ayurveda have developed a variety of herbal therapeutic modalities for acne. This improvement may be attributed to various pharmacological activities of the ingredients of PHUF (Zimade Mohasa). These herbs possess its ingredients are irsa (Iris florentina), barghe neem (Azadirachta indica leaves), poste saras (Acacia speciosa bark), ghungchi safaid (Abrus precatorious) and namake sambhar (lake salt) 50 grams each [8]. Zimade mohasa acts topically as a detergent, astringent, anti-inflammatory and antibacterial agent [9].

Homeopathic medicine has shown a significant popularity among people seeking treatment for acne. In an Indian study of 400 patients with acne, significant improvement was seen in those who were started on oral homeopathic medicine (sulphur or tuberculinum) as compared to the control group. Homeopathic products such as lycopodium, sepia, staphisagria, lachesis and nat-m have also been successfully used in patients with acne [10]. Arctium Lappa with its active ingredients present in the root namely, inulin, poly-acetylenes, tannin, lignans, arctigenin has shown significant improvement in acne. These have been found to promote blood circulation and have anti-inflammatory effect [11].

Biofeedback and cognitive therapy have also found a role in treatment of acne. A number of studies have suggested the role of psychological stress in pathogenesis of acne by modulation of pituitary hypothalamic axis and thyroid and sex hormones. It is proposed that muscle relaxation and cognitive therapy can therefore aid in acne management [10-12].

Naturopathic modalities like therapeutic fasting and yoga have 
also been used in the treatment of acne vulgaris. According to the naturopathic medicine, body has its own power to heal itself and dietary changes and yoga have direct influence on the skin diseases like acne by modulation of inflammatory cytokines $[6,13,14]$.

\section{Conclusion}

Various complementary and alternative medicine modalities have been introduced and found to have an important role in management of acne where the conventional and standard treatment cannot be utilized. However, their recommendation for treatment is limited by lack of large scale studies and evidence about their efficacy and safety profile.

\section{References}

1. Ahmad A, Alghanemi L, Alrefaie S, et al. The use of complementary medicine among acne valguris patients: Cross sectional study. J Dermatology Dermatologic Surg 2017; 21: 66-71.

2. Pond DC, Smith W, Heading GS et al. Complementary and alternative medicine therapies in acne, psoriasis, and atopic eczema: Results of a qualitative study of patients' experiences and perceptions. J Altern Complement Med 2006; 12: 451-457.

3. Wysong A, Higgins S, Ahadiat $\mathrm{O}$, et al. Complementary and alternative medicine (CAM) and acne. J Am Acad Dermatol 2017; 76: 81 .

4. Teoh SM, Xi SY, Wang YH, et al. Comprehension and experience of acne treated with traditional chinese medicine facial mask of bai zhi (Radix Angelicae Formosanae) and bai fu zi (Rhizoma Typhonii Gigantei) as the basis formula by external application. Chin Med 2012; 3: 87-93.

5. Ghosh VK, Nagore DH, Kadbhane KP, et al. Different approaches of alternative medicines in acne vulgaris treatment. Orient Pharm Exp Med 2011; 11: 1-9.

6. Magin P, Adamas J, Heading G, et al. Complementary and alternative medicine therapies in acne, psoriasis, and atopic eczema. J Altern Complement Med 2006; 12: 451-457.

7. Cao H, Yang G, Wang Y, et al. Acupoint stimulation for acne : A systematic review of randomized controlled trials. Med Acupunct 2013; 25: 173-195.

8. Xu J, Lin R, Wang J, et al. Effect of acupuncture anesthesia on acne vulgaris of pricking-blood-letting cupping: A single-blind randomized clinical trial. J Tradit Chinese Med 2013; 33: 752-756.

9. Lone AH, Habib S, Ahmad T, et al. Effect of a polyherbal unani formulation in acne vulgaris: A preliminary study. $\mathrm{J}$ Ayurveda Integr Med 2012; 3: 180-183.

10. Itamura R. Effect of homeopathic treatment of 60 Japanese patients with chronic skin disease. Complement Ther Med 2007; 15: 115-120.

11. Miglani A, Manchanda RK. Observational study of Arctium lappa in the treatment of acne vulgaris. Homeopathy 2014; 103: 203-207.

12. Zaenglein AL, Pathy AL, Schlosser BJ, et al. Guidelines of care for the management of acne vulgaris. J Am Acad Dermatol 2016; 74: 945-973.

13. Ameya P, Nair PMK. Role of therapeutic fasting along with other naturopathy and yoga modalities in addressing acne vulgaris-A single case report. J Fasting Health 2017; 5: $103-106$.

14. Cao H, Yang G, Wang Y, et al. Complementary therapies for acne vulgaris. Cochrane Database Syst Rev 2016; 1: 138.

\section{*Correspondence to}

Ishmeet Kaur,

Senior Resident,

Department of Dermatology \& STD,

University College of Medical Sciences \& GTBH,

New Delhi 110095, India.

Tel: +91 9873771701

E-mail: ishmeet.kaur.dr@gmail.com 